\title{
Towards a cure for HIV: the identification and character- ization of HIV reservoirs in optimally treated people
}

\author{
Adewunmi Onafuwa-Nuga ${ }^{1}$, Lucy A McNamara ${ }^{2,3}$, Kathleen L Collins ${ }^{1,2}$ \\ ${ }^{I}$ Department of Internal Medicine, University of Michigan Medical School, Ann Arbor, Michigan 48109, USA; ${ }^{2}$ Department of Mi- \\ crobiology and Immunology, University of Michigan Medical School, Ann Arbor, Michigan 48109, USA; ${ }^{3}$ Department of Epidemi- \\ ology, University of Michigan, Ann Arbor, Michigan 48109, USA
}

Currently available anti-HIV-1 drugs suppress viral replication and maintain viral levels below the detection threshold of most assays but do not eliminate cellular reservoirs. As a result, very low levels of circulating virus can be detected in most people despite long-term treatment with potent anti-HIV drug combinations. Not surprisingly, viral levels rebound with discontinuation of treatment. New evidence indicates that there is a viral reservoir in bone marrow progenitor cells.

Keywords: HIV; latency; bone marrow; stem cells; CD34

Cell Research (2010) 20:1185-1187. doi:10.1038/cr.2010.140; published online 28 September 2010

Over ten years ago, it was demonstrated that HIV establishes cellular reservoirs of latent virus in resting $\mathrm{CD}^{+} \mathrm{T}$ cells [1]. However, subsequent studies in which residual viral genomes were sequenced and compared to cellular proviral sequences suggested that reactivation of latently infected $\mathrm{CD} 4^{+} \mathrm{T}$ cells could not account for the majority of the residual virus [2,3]. Recent studies of viral load decay kinetics after the initiation of highly active antiviral therapy have also suggested the existence of additional reservoirs, including at least one reservoir that did not appreciably decay after seven years [4]. The identification and characterization of HIV-1 cellular reservoir(s) in effectively treated patients (HIV-1 RNA below 50-75 copies) is now a high priority as a first step towards the development of a strategy for virus eradication.

Recently, several studies have demonstrated that multipotent hematopoietic stem/precursor cells (HSPCs) can become infected with HIV-1 [5, 6]. Moreover, results from one study suggest that these cell types may be an important cellular reservoir for HIV-1 [5].

Most multipotent hematopoietic precursor cells express CD34, a cell-surface marker found on HSPCs rang-

Correspondence: Kathleen L Collins

Tel: 734-615-1320; Fax: 734-615-5252

E-mail: klcollin@umich.edu ing from hematopoietic stem cells (HSCs) to progenitor cells committed to differentiation. $\mathrm{CD} 34^{+}$populations that lack CD38 and express CD133 are enriched for multipotent progenitor cells. However, functional assays demonstrating the capacity of cells to differentiate are necessary to definitively identify multipotent cells. Multipotent cells (HSCs, multipotent progenitor cells (MPPs), and common myeloid progenitor cells (CMPs)) are capable of forming colonies containing cells of all myeloid lineages in methylcellulose. Because lymphoid cells do not grow under these conditions, these assays do not distinguish HSCs and MPPs from CMPs.

A proportion of $\mathrm{CD} 34^{+}$cells express the HIV receptors CD4, CXCR4, and CCR5, making these cells potentially susceptible to HIV-1 infection (reviewed in [7]). Beginning more than 20 years ago, some studies suggested that rare infection of $\mathrm{CD} 34^{+}$cells could occur both in vitro and in vivo [8-11]; however, the authors could not rule out the possibility of contamination by other cell types. Furthermore, studies assessing HIV-1 infection of multipotent colony-forming or $\mathrm{CD} 133^{+} \mathrm{HSPCs}$ failed to detect either HIV-1 infection or expression of CD4, CXCR4 and CCR5 in these cells [12-14].

Reexamination of this topic has provided important new evidence that a percentage of multipotent HSPCs are susceptible to HIV infection. A 2007 study examined the ability of HIV-1 subtype C to infect multipotent CD34 HSPCs in vitro and in vivo [6]. The authors found that 
several isolates of HIV-1C could infect multipotent cells in vitro and that HIV was detected in most (12 of 19) CD34 ${ }^{+}$cell samples isolated from HIV-infected donors with AIDS. Moreover, levels of provirus in the CD34 cells were higher than levels observed in total peripheral blood mononuclear cells in 11 of the 12 with detectable provirus in their CD34 $4^{+}$HSPCs. Notably, these authors could not detect HIV DNA in multilineage colonies generated from HSPCs exposed to HIV-1B isolates - however the authors could not rule out the possibility that HIV-1B infection is cytotoxic leading to cell death rather than infected colony formation.

To further explore HIV-1B infection of HSPCs, we used flow cytometric approaches to detect the expression of the HIV-1 Gag protein in CD34 ${ }^{+}$cells after very short incubation periods (three days) [5] and demonstrated that five full-length viruses had the capacity to infect CD $34^{+}$ cells. Notably, the use of flow cytometry avoided potential contamination problems that plagued prior studies because it allowed the simultaneous examination of individual cells for expression of both CD34 and viral proteins. Moreover, the use of five different full length live viruses (some of which were initially isolated directly from patient samples) demonstrated the physiological relevance of this result.

Intracellular Gag staining allows simultaneous analysis of multiple markers on individual cells, but there is potential for false positive results. However, HIV-1 Gag expression was dramatically reduced by pre-treatment of the cells with anti-retroviral drugs such as AZT [5]. $\mathrm{Gag}^{+}$ cells could be detected in freshly isolated cells from some donors with high viral loads but not in cells from donors optimally treated with antiretroviral drugs [5]. Additionally, Gag-positive cells expressed functional HIV Nef based on their ability to downmodulate MHC-I $[15,16]$. Similar results were obtained using reporter viruses that expressed marker genes such as GFP or placental alkaline phosphatase. Infection was further confirmed by the detection of viral particles released from $\mathrm{CD} 34^{+}$cells, by the demonstration that viral particles released from CD $34^{+}$cells had the capacity to infect $\mathrm{T}$ cells and by the detection of integrated viral genomes in $\mathrm{CD}^{+} 4^{+}$cells [5]. Based on these controls and confirmatory experiments, Gag staining represented bona fide infection.

All five of the full length HIV-1s we tested in vitro were cytopathic and the number of infected CD $34^{+}$cells declined dramatically over time with continued cell culture. Therefore to measure the capacity of infected cells to form mutilineage colonies, $\mathrm{CD} 34^{+}$cells were infected with a non-cytotoxic, GFP-expressing HIV-1B that lacked expression of potentially toxic viral gene products [5]. Using these conditions, we could detect multilineage colonies that were uniformly $\mathrm{GFP}^{+}$, demonstrating that HIV-1B envelopes can target multipotent HSPCs and that HIV integration can occur in multipotent HSPCs in vitro. However, this assay is not able to determine whether these cells were HSCs, MPPs and/or CMPs.

Importantly, HIV could establish latency in HSPCs infected in vitro. After the initial infection had declined and active infection could no longer be observed, agents that stimulated myeloid differentiation resulted in a resurgence of viral gene expression [5]. This observation was easiest to demonstrate using viruses that were able to amplify the signal by spreading infection to the differentiating macrophages, but we were able to detect reactivation of virus even in the presence of antiretroviral drugs that blocked viral spread [5]. We also developed an HIV that allowed us to directly detect a population of latently infected cells $\left(\mathrm{GFP}^{+}, \mathrm{HIV} \mathrm{Gag}{ }^{-}\right)$that was stable in culture [5]. Notably, some latently infected cells were $\mathrm{lin}^{-}, \mathrm{CD} 38^{-}$and CD $34^{+}$, consistent with multipotent cells. Together, these data provide evidence that latent HIV-1 infection of primitive HSPCs is possible in vitro.

To determine whether HIV infects $\mathrm{CD} 34^{+}$bone marrow HSPCs in vivo, we obtained bone marrow from $\mathrm{HIV}$-infected individuals. Three out of six $\mathrm{HIV}^{+}$individuals with high viral loads $(>50000$ copies $/ \mathrm{ml}$ ) had detectable $\mathrm{Gag}^{+} \mathrm{CD} 34^{+}$(and $\mathrm{CD} 34^{-}$) cells. The use of flow cytometry was crucial for these studies because Gag could be detected within both CD $34^{+}$and CD $34^{-}$ cells in this cohort. In contrast, $\mathrm{CD} 34^{+}$cells from donors with clinically undetectable viral loads for more than six months did not express detectable amounts of HIV Gag. In the three high viral load donors that were initially negative for $\mathrm{Gag}^{+} \mathrm{CD} 34^{+}$cells and in one donor with undetectable viral load, HIV Gag expression was induced upon myeloid differentiation of CD34 ${ }^{+}$. This finding provides evidence that $\mathrm{CD} 34^{+}$cells could also harbor latent HIV in some donors, even those with clinically undetectable viral loads for more than six months on optimal therapy.

To examine whether $\mathrm{CD} 34^{+}$cells harboring HIV genomes persisted after more than six months of optimal therapy, $\mathrm{CD} 34^{+}$bone marrow cells were obtained from optimally treated individuals with clinically undetectable viral loads ( $<48$ copies $/ \mathrm{ml})$. As expected, optimally treated donors had no evidence of Gag expression in freshly isolated CD $34^{+}$cells. However, HIV-1 DNA could be amplified from $\mathrm{CD} 34^{+}$cells from more than $40 \%$ of these donors. In contrast, we were unable to detect comparable amounts of HIV DNA from bone marrow cells depleted for $\mathrm{CD} 34^{+}$cells, indicating that infected CD34- bone marrow cells do not persist at similar levels in people on HAART [5]. The ability of $\mathrm{CD} 34^{+}$cells to 
harbor latent HIV in vivo, even in patients on optimal therapy, indicates that $\mathrm{CD} 34^{+}$cells can act as a long-lived reservoir of HIV.

Additional studies are now needed to clarify which subsets of $\mathrm{CD}_{3} 4^{+}$cells harbor HIV genomes in vivo and whether latently infected $\mathrm{CD} 34^{+}$cells contribute to residual viremia in patients on HAART. More research will also be needed to determine whether different types of HIVs target different subsets of CD $34^{+}$cells.

For many years it has been unclear whether multipotent HSPCs could be infected with HIV-1. Recent studies have now provided evidence that both active and latent infection can happen in vitro and in vivo. Infection of these HSPCs may contribute to hematopoietic defects noted in late stage HIV disease. Moreover, latent infection of HSPCs may create a stable long-lived reservoir that contributes to residual plasma viremia in optimally treated patients. Successful eradication of HIV will require clearance of virus from all cellular reservoirs.

\section{Acknowledgments}

Funding for this work was received from the National Institutes of Health and the Burroughs Wellcome Fund. LM is a recipient of an NSF pre-doctoral fellowship and a Bernard Maas Fellowship.

\section{References}

1 Finzi D, Hermankova M, Pierson T, et al. Identification of a reservoir for HIV-1 in patients on highly active antiretroviral therapy. Science 1997; 278:1295-1300.

2 Bailey JR, Sedaghat AR, Kieffer T, et al. Residual human immunodeficiency virus type 1 viremia in some patients on antiretroviral therapy is dominated by a small number of invariant clones rarely found in circulating CD4+ T cells. J Virol 2006; 80:6441-6457.

3 Brennan TP, Woods JO, Sedaghat AR, Siliciano JD, Siliciano RF, Wilke CO. Analysis of human immunodeficiency virus type 1 viremia and provirus in resting CD4+ T cells reveals a novel source of residual viremia in patients on antiretroviral therapy. J Virol 2009; 83:8470-8481.
4 Palmer S, Maldarelli F, Wiegand A, et al. Low-level viremia persists for at least 7 years in patients on suppressive antiretroviral therapy. Proc Natl Acad Sci USA 2008; 105:38793884.

5 Carter CC, Onafuwa-Nuga A, McNamara LA, et al. HIV1 infects multipotent progenitor cells causing cell death and establishing latent cellular reservoirs. Nat Med 2010; 16:446451.

6 Redd AD, Avalos A, Essex M. Infection of hematopoietic progenitor cells by HIV-1 subtype C, and its association with anemia in southern Africa. Blood 2007; 110:3143-3149.

7 Alexaki A, Wigdahl B. HIV-1 infection of bone marrow hematopoietic progenitor cells and their role in trafficking and viral dissemination. PLoS Pathog 2008; 4:e1000215.

8 Folks TM, Kessler SW, Orenstein JM, Justement JS, Jaffe ES, Fauci AS. Infection and replication of HIV-1 in purified progenitor cells of normal human bone marrow. Science 1988; 242:919-922.

9 Stanley SK, Kessler SW, Justement JS, et al. CD34+ bone marrow cells are infected with HIV in a subset of seropositive individuals. J Immunol 1992; 149:689-697.

10 Davis BR, Schwartz DH, Marx JC, et al. Absent or rare human immunodeficiency virus infection of bone marrow stem/ progenitor cells in vivo. J Virol 1991; 65:1985-1990.

11 Neal TF, Holland HK, Baum CM, et al. CD34+ progenitor cells from asymptomatic patients are not a major reservoir for human immunodeficiency virus-1. Blood 1995; 86:17491756.

12 Chelucci C, Hassan HJ, Locardi C, et al. In vitro human immunodeficiency virus-1 infection of purified hematopoietic progenitors in single-cell culture. Blood 1995; 85:1181-1187.

13 Shen H, Cheng T, Preffer FI, et al. Intrinsic human immunodeficiency virus type 1 resistance of hematopoietic stem cells despite coreceptor expression. J Virol 1999; 73:728-737.

14 Weichold FF, Zella D, Barabitskaja O, et al. Neither human immunodeficiency virus-1 (HIV-1) nor HIV-2 infects mostprimitive human hematopoietic stem cells as assessed in longterm bone marrow cultures. Blood 1998; 91:907-915.

15 Collins KL, Chen BK, Kalams SA, Walker BD, Baltimore D. HIV-1 Nef protein protects infected primary cells against killing by cytotoxic T lymphocytes. Nature 1998; 391:397-401.

16 Schwartz O, Marechal V, Le Gall S, Lemonnier F, Heard JM. Endocytosis of major histocompatibility complex class I molecules is induced by the HIV-1 Nef protein. Nat Med 1996; 2:338-342. 